\title{
Barriers of Occupation-based Intervention
}

\author{
Ahmad Zamir Che Daud ${ }^{1,2}$, Jenni Judd¹, Matthew Yau ${ }^{1}$, Fiona Barnett ${ }^{1}$ \\ 1 Division of Tropical Health and Medicine, James Cook University Australia \\ 2 Department of Occupational Therapy, Faculty of Health Sciences, Universiti Teknologi Mara Puncak Alam \\ zamir_1225@yahoo.com
}

\begin{abstract}
A Delphi study with three rounds of inquiry was conducted to identify the barriers of implementing Occupationbased Intervention $(\mathrm{OBI})$ in Malaysia. Fifteen occupational therapy practitioners and educators consented and completed all the Delphi rounds. The first Delphi round began with an open-ended questionnaire asking the participants a broad question on barriers for applying $\mathrm{OBI}$ into clinical practice. Data was qualitatively analysed to develop statements about the barriers of applying $\mathrm{OBI}$ were grouped under five categories. In the second and third round, the participants were asked to rank their agreement with the statements about the barriers in applying OBI. Twenty-seven statements finally achieved the consensus level.
\end{abstract}

Keywords: Occupation, Clinical Practice, Occupational Therapy, Service Delivery

eISSN: 2398-4279 @ 2016. The Authors. Published for AMER ABRA by e-International Publishing House, Ltd., UK. This is an open access article under the CC BY-NC-ND license (http://creativecommons.org/licenses/by-ncnd/4.0/). Peer-review under responsibility of AMER (Association of Malaysian Environment-Behaviour Researchers), ABRA (Association of Behavioural Researchers on Asians) and cE-Bs (Centre for EnvironmentBehaviour Studies), Faculty of Architecture, Planning \& Surveying, UniversitiTeknologi MARA, Malaysia.

https://doi.org/10.21834/ajqol.v1i4.12 


\subsection{Introduction}

Since the early 20th century, occupational therapists have been using occupation as their primary intervention medium for people with mental illness and physical disability. The development of the profession was influenced by the moral treatment that prevailed in the $18^{\text {th }}$ and $19^{\text {th }}$ centuries as a therapy for people who were mentally ill. The assumption of the moral treatment was that engagement in various daily occupations could restore the individual's health and functioning (Keilhofner, 2009). Occupation includes: Activities of Daily Living (ADLs), Instrumental Activities of Daily Living (IADLs), work, education, play, leisure, rest and sleep, and social participation (American Occupational Therapy Association, 2014). Occupational therapists continued using occupation as the core therapeutic means of intervention until the profession was pressured by the modern Biomedical Model that insisted on providing rationale for practice, which led to development of the mechanistic paradigm (Keilhofner, 2009). The influence of this mechanistic paradigm had caused occupational therapists to distance themselves from using occupation and its more holistic process to the mechanistic paradigm that tends to focus on understanding and addressing body functions and impairments (Keilhofner, 2009).

Malaysian occupational therapist perceived OBI as a means and as an end (Che Daud, Yau, \& Barnett, in press). Occupation as a means refers to occupation as an agent to improve impaired function, while occupation as an end refers to occupation to be accomplished by the clients (Gray, 1998; Trombly, 1995). The benefits of OBI have been well documented in the literature. For instance, a recent study found $\mathrm{OBI}$ was effective in improving ADLs and quality of life for the clients with stroke (Shinohara, Yamada, Kobayashi, \& Forsyth, 2012). Although OBI benefits the clients, there are challenges for many occupational therapists to use $\mathrm{OBI}$ within their practice context, particularly for those who are working in the medically-oriented facilities. This study aimed to identify barriers in applying $\mathrm{OBI}$ in clinical practice.

\subsection{Literature Review}

Several challenges in applying $\mathrm{OBI}$ were identified in literature. One of these challenges is the dominance of the Biomedical Model in health care practice (Colaianni \& Provident, 2010). This mechanistic paradigm that was derived from the Biomedical Model had diverted the central idea of the profession, which was to concentrate on occupation as a healthrestoring measure and to focus on remediation of body functions and impairments (Keilhofner, 2009). As the Biomedical Model mainly focused on curing disease by reducing impairments and eliminating symptoms, practising occupational therapy in medicallyoriented facilities led to the impairment-based treatment practice where the body functions and impairments became the primary outcome of the intervention (Gray, 1998). It is also difficult to incorporate health, wellness and functions within the medical paradigm of care (Baum \& Baptise, 2002). For instance, occupational therapists found it was difficult to fit 
occupations such as play, cooking, craft, self-care routine and pleasurable activities within the biomedical model dominated setting as it was felt that these tasks were not scientific enough to address body functions and impairments of the clients (Burke, 2001). As a result, occupational therapists tended to neglect the use of occupation in practice, which may have indirectly contributed to the profession struggling with professional identity (Golledge, 1998a; Gray, 1998).

Lack of facilities was another barrier in applying $\mathrm{OBI}$ in clinical practice. A study conducted by Stack and Barker (2011) found that the occupational therapy students would eagerly translate $\mathrm{OBI}$ in practice setting, but environmental factors prevented them from doing that. Limited space and the availability of equipment and supplies were the main barriers highlighted by occupational therapists in their use of $\mathrm{OBI}$ (Chisholm, Dolhi, \& Schreiber, 2004). As the settings are built up in the medical-oriented facilities, most of the available equipment focuses on remediating impairments and body functions. Pragmatically, occupational therapists often use what is typically available in the department or clinical setting (Gray, 1998). Equipping a department that is suitable for OBI requires funding from the organisation. However, not all organizations could provide the money or the equipment, and these supplies are lacking in the practice setting (Chisholm et al., 2004).

Time was also a factor that influences the occupational therapists to use OBI. Literature indicates that $\mathrm{OBI}$ was described as too complicated and consumes much time to be implemented (Goldstein-Lohman, Kratz, \& Pierce, 2003; Stack \& Barker, 2011). Occupational therapists also agree that they could do more for the clients. However, addressing each client's occupational needs takes more time and results in another client not receiving an intervention in a timely manner (Stack \& Barker, 2011). Occupational therapists who are practicing in an acute setting, such as in hand injury rehabilitation are expected to provide impairment-based treatment instead of OBI. Given the high volumes of caseload and the role expectations in the clinical practice setting, occupational therapists tend to see time as a major barrier to using OBI (Colaianni \& Provident, 2010; Stack \& Barker, 2011).

The issue of reimbursement is also a barrier to applying $\mathrm{OBI}$. For instance, occupational therapists in the United States reported that $\mathrm{OBI}$ was not covered by insurance companies and documenting the intervention for billing purposes was difficult (Colaianni \& Provident, 2010). Rogers (2007) described that billing for $O B I$ is not straightforward and that occupational therapists have to explain to the insurance companies why they have provided the clients with that specific intervention. As a result, there is a lack of opportunity for the occupational therapists to explore the occupational performance problems and to identify the barriers to successful occupational performance within the client's context (Toth-Fejel, Toth-Fejel, \& Hedricks, 1998).

Another challenge to $\mathrm{OBI}$ in the literature was the client and occupational therapist factors. Occupational therapists reported that providing $\mathrm{OBI}$ is challenging because the client does not understand the unique role of occupational therapy nor the outcome of the 
intervention (Chisholm et al., 2004). From occupational therapists' perspectives, they have much less understanding of the environmental concept of objects, spaces and occupational forms, which contribute to the challenge of using OBI (Lee, Taylor, Kielhofner, \& Fisher, 2008). Providing $\mathrm{OBI}$ requires the occupational therapist to know more about the client's context and to use appropriate interventions to achieve occupational performance goals. Additionally, occupational therapists often undermined the value and power of occupation in rehabilitating the clients (Chisholm et al., 2004). They think that occupation cannot meet the client's goal, and is unnecessary and too complicated for the clients (Colaianni \& Provident, 2010).

Most Malaysian occupational therapists are working in medically-oriented settings such as hospitals and health clinics, where the provision of $\mathrm{OBI}$ is challenging (Aiken, Fourt, Cheng, \& Polatajko, 2011). Given there are no published studies conducted in Malaysia regarding this issue, the aim of this study was to identify barriers in applying $\mathrm{OBI}$ in clinical practice.

\subsection{Methodology And Results}

\subsection{Design}

A Delphi technique with three rounds of inquiry was conducted to identify the challenges of applying $\mathrm{OBI}$ in Malaysian occupational therapy practice. This technique is efficiently used when there are contradictory and insufficient facts about a certain issue (Hasson, Keeney, \& McKenna, 2000). The Delphi technique is a mixed method approach, and often begins with qualitative data collection and is followed by quantitative data collection (Keeney, Hasson, \& McKenna, 2001). There are three types of Delphi technique, namely conventional, real-time and policy (De Villiers, De Villiers, \& Kent, 2005). This study employed the conventional Delphi technique, in which an open-ended questionnaire was sent to a group of experts in the first round and then followed by a close-ended questionnaire in the subsequent rounds. This technique was used because there is insufficient information about the challenges encountered by Malaysian occupational therapists in applying $\mathrm{OBI}$. Due to the nature of Delphi technique, which involves an iterative process, it is hard to separate the methods and results in different sections. This study was approved by the Human Research Ethics Committee of James Cook University (H4559), Institute of Public Health Malaysia (NMRR-12-53-10918) and the Economic Planning Unit Malaysia (UPE:40/200/19/2865).

\subsection{Participants}

This study used purposive sampling to select expert occupational therapists in Malaysia. According to Malaysian Occupational Therapist Association (MOTA) (2010), there were 213 occupational therapists employed within Ministry of Health Malaysia (MOH) facilities and 
most of them were based in the public hospitals. An expert, as defined in this study, is a person who holds a senior position at work (Kuipers \& Grice, 2009) and was nominated by peers to have pertinent expertiseness in certain areas of professional specialty (Jensen, Gwyer, \& Shepard, 2000). Additionally, Unsworth (2001) differentiated the novice and expert occupational therapist by years of experience, where the experts were described as having at least five years' experience in the occupational therapy field. Therefore, the inclusion was set as: (i) occupational therapy practitioners and educators who are holding a senior position; (ii) qualified at least with a bachelor's degree in occupational therapy; and (3) have at least 5 years' experience in the field of occupational therapy practice. Initially, lists of occupational therapy practitioners and educators who met the inclusion criteria were obtained from Head of Malaysia Occupational Therapy Service and President of MOTA. A simple survey was conducted by inviting occupational therapists from various backgrounds to nominate the occupational therapy experts in Malaysia. A list was compiled, consisting of 52 eligible occupational therapy practitioners and educators. An invitation letter was sent to all eligible participants in the middle of June 2012. Fifteen occupational therapy practitioners and educators consented to take part in the study. All the participants completed all Delphi rounds. Most of the participants were senior occupational therapy practitioners $(n=8)$ and educators $(n=7)$ with more than five years' experience in clinical practice. The range of the participant's age was between 29 to 49 years old. Ten of the participants were qualified with a Bachelor's Degree, three with a Master's Degree and two with a Doctor of Philosophy.

\subsection{Round One}

In early July 2012, the first questionnaire's link was sent to all participants who consented to take part in the study. It was an open-ended questionnaire that asked the participants a broad question about the challenges of applying OBI in clinical practice. The function of the first round was to identify the issues that could be brought forward to later rounds and used open-ended questions to increase the richness of the data collected (Powell, 2003). Additionally, participants' demographic details such as gender, age, professional qualifications, position and clinical experience were obtained during this Delphi round. The responses were analysed using simplified Colaizzi's thematic analysis (Creswell, 2007). Five categories emerged from the analysis of first round questionnaire namely: the client factors, occupational therapist factors, contextual factors, occupation as treatment modalities and logistic issues. Forty-two statements were developed under these categories to formulate the second round questionnaire.

\subsection{Round Two}

At the end of August 2012, the second round questionnaire's link was sent to the participants. They were asked to rank their agreement with the statement generated from the data collected in the first round using four-point Likert type scale from: (1) totally disagree; (2) disagree; (3) agree; and (4) totally agree (Mullersdorf \& Ivarsson, 2011), on the issues in applying $\mathrm{OBI}$. The participants were also allowed to comment on the 
statements and add new information about the issue studied. Data was analysed using IBM SPSS version 20.0 by calculating the percentage of agreement for each statement. The level of consensus was set at $\geq 70 \%$, which meant that two-thirds of the participants must agree to strongly agree with each of statements (Keeney, Hasson, \& McKenna, 2006; Mullersdorf \& Ivarsson, 2011). The comments and new information from participants were analysed and synthesised to improve the statements or if appropriate to develop new statements. Analysis of the second round survey found that eighteen out of forty-two statements achieved the pre-consensus level. Two participants added new information, which led to the development of two new statements.

\subsection{Round Three}

In the middle of October 2012, the link of the third round survey was sent to all participants. The participants again were asked to rank their agreement to achieve the final consensus about the statements on challenges of using OBI but were limited to agree or disagree. The level of consensus was set the same as the second round. An analysis of the second round survey was also supplied to help participants refine their responses. A comment box was provided at the end of the survey for participants to comment and add new information about the barriers of applying OBI. Nine more statements achieved the pre-set consensus level in the third round. Total statements that achieved pre-set consensus were twentyseven. Seventeen statements did not achieve the pre-set consensus level.

\subsection{Discussions}

This study found several barriers to $\mathrm{OBI}$ in the Malaysian context. However, the findings reflected the common challenges to $\mathrm{OBI}$ regardless of practice area. One of the challenges came from the client factors. This study supports the previous findings that the client does not understand the purpose of $\mathrm{OBI}$, which contributed to the challenges of applying the intervention in a practice context (Chisholm et al., 2004; Colaianni \& Provident, 2010). Furthermore, the clients also have no idea about the unique role of occupational therapy and are not aware of the effect of $\mathrm{OBI}$ (Chisholm et al., 2004). The client's understanding of the whole recovery process was also cited as a challenge to $\mathrm{OBI}$. Another challenge from the clients was that they were more impressed and motivated by sophisticated and advanced equipment. In contrast, $\mathrm{OBI}$ only uses materials related to the client's occupation.

Barriers to $\mathrm{OBI}$ also came from the occupational therapists themselves (occupational therapy factors). Participants described that they were not trained or well prepared for OBI. In fact, they have limited knowledge and understanding about OBI. Occupational therapists who used impairment-based treatment usually was influenced by previous training and clinical experience (Goldstein-Lohman et al., 2003). They also agreed that they lacked skills in grading and analysing activities, which is an important aspect of providing OBI. The participants perceived that they also lacked the creative skills to implement OBI. These 
results support the statement that the credibility of occupational therapists is the challenge to OBI (Colaianni \& Provident, 2010).

Providing $\mathrm{OBI}$ required the occupational therapist to know the client's occupation, motivation and life situation (Baum, 2000), which only can be achieved through the use of a client-centred approach and working partnership with the clients (Baum \& Baptise, 2002; Chisholm et al., 2004). However, the majority of participants admitted that Malaysian occupational therapists rarely use the client-centred approach in practice, which is a challenge to $\mathrm{OBI}$. The perceived challenge was related to the skills and knowledge lacking in applying the client-centred approach among the occupational therapists. Additionally, most of the participants admitted that they rarely use occupation-based assessment in practice. Therefore, the intervention is not focused on settling the client's occupational performance needs.

This study also identified few contextual factors that restrict occupational therapists to provide OBI. First was the Malaysian cultural value, where the family members feel obligated to help the clients in their daily occupations. That's the challenge I often face". It has been reported that families of Asian clients tend to be overinvolved, and even might take over the client's responsibilities and make decisions on behalf of the clients (Nilchaikovit, Hill, \& Holland, 1993). A second factor was the health care system and government policy in Malaysia (Chisholm et al., 2004). The participants described that the medically-oriented health care services and the bureaucratic culture within Malaysia hospitals were also challenges to OBI. The ultimate treatment goal is to cure the impairments without considering other issues, and the occupational therapist is obligated to follow the doctor's instruction as they are higher in the health professional hierarchy in Malaysia.

Another barrier to OBI within medical-oriented facilities was the health professionals' view about the diagnosis. For instance, a particpant in this study stated that "the multidisciplinary members always perceive movement and strength are the main requirements for function". Other elements such as the client's ability to perform daily occupations and how the client's context affects occupational performance are often neglected. Furthermore, lack of awareness about the role of occupational therapists by other professionals was perceived as a challenge to $\mathrm{OBI}$ as this limits the referrals for the intervention. Most of the participants stated that when they try to use $\mathrm{OBI}$ in practice, other multidisciplinary professionals do not give full cooperation because they do not understand the purpose of OBI (Chisholm et al., 2004; Colaianni \& Provident, 2010).

The credibility of occupation as a treatment modality was also perceived by the participants as a challenge to $\mathrm{OBI}$. They asserted that there was limited evidence on the efficacy of OBI to support their practice (Colaianni \& Provident, 2010). Furthermore, occupational therapists think that certain $\mathrm{OBI}$ is not practical for the acute setting. Additionally, occupations as the ultimate goal are hard to achieve and complex to measure (Coster, 2008). 
Logistics issues also influence the capability of occupational therapists to provide $\mathrm{OBI}$. Time and workload were reported as challenges to implementing the intervention (Colaianni \& Provident, 2010; Stack \& Barker, 2011). The participants claimed that practising OBI consumed more time, but they do not have time for that because of the high volume of caseloads per day. Guidelines about OBI from the MOTA also were not available for occupational therapists to make proper referrals. Logistic issues such as resources, equipment, and the environmental context were also reported as barriers to $\mathrm{OBI}$ (Chisholm et al., 2004; Colaianni \& Provident, 2010; Goldstein-Lohman et al., 2003). The majority of participants agreed that appropriate resources and equipment are lacking, and the occupational therapy department is not set up for OBI. Finally, the available resources and equipment were mainly for impairment-based treatment.

\subsection{Conclusion}

This study identified the challenges encountered by occupational therapists in applying $\mathrm{OBI}$ into the Malaysian practice context. The client and occupational therapist factors, logistic issues, the credibility of occupation and contextual factors are challenges to implementing $\mathrm{OBI}$ in clinical practice. These challenges need to be solved to provide better treatment outcomes for the client where $\mathrm{OBI}$ helps to enhance the quality of life following a health event or disease. Reflective practice, research, education, promotion, and training may be the potential solutions to these issues. Applying the adoption of $\mathrm{OBI}$ into practice creates occupational therapy as a unique, holistic and more client-centred approach. Additionally, practising $\mathrm{OBI}$ allows occupational therapists to maintain their unique identity, which is centered on occupation to promote health, wellbeing and quality of life. Further qualitative research is needed to explore the experiences of occupational therapists providing $\mathrm{OBI}$ in a specific area of practice especially, in an acute setting such as hand rehabilitation.

\section{Acknowledgement}

The authors would like to thank all participants for their time and commitment. This study was funded by the Division of Tropical Health and Medicine, James Cook University, Australia.

\section{References}

Aiken, F. E., Fourt, A. M., Cheng, I. K., \& Polatajko, H. J. (2011). The meaning gap in occupational therapy: Finding meaning in our own occupation. Canadian Journal of Occupational Therapy, 78(5), 294-302.

American Occupational Therapy Association. (2014). Occupational therapy practice framework: Domain and process, third edition. American Journal of Occupational Therapy, s1-s48. 
Che Daud, A.Z., et.al., / Asian Journal of Quality of Life, AjQoL, 1(4), Nov. / Dec. 2016 (p.1-10)

Baum, C. (2000). Occupation-based practice: Reinventing ourselves for the new millennium. OT Practice, 5(1), 1215.

Baum, C. M., \& Baptise, S. (2002). Reframing occupational therapy practice In M. Law, C. M. Baum \& S. Baptise (Eds.), Occupation-based practice fostering performance and participation (pp. 3-16). Thorofare: Slack Incorporated

Burke, J. P. (2001). How therapists' conceptual perspectives influence early intervention evaluations. Scandinavian Journal of Occupational Therapy, 8(1), 49-61.

Che Daud, A. Z., Yau, M., \& Barnett, F. (in press). A consensus definition of occupation-based intervention from a Malaysian perspective: A Delphi study. British Journal of Occupational Therapy.

Chisholm, D., Dolhi, C., \& Schreiber, J. (2004). Occupational therapy intervention resource manual: A guide for occupation-based practice. Australia: Thomson Delmar Learning.

Colaianni, D., \& Provident, I. (2010). The benefits of and challenges to the use of occupation in hand therapy. Occupational Therapy in Health Care, 24(2), 130-146.

Coster, W. J. (2008). 2008 Eleanor Clarke Slagle Lecture. Embracing ambiguity: Facing the challenge of measurement. American Journal of Occupational Therapy, 62(6), 743-752.

Creswell, J. W. (2007). Qualitative inquiry and research design: Choosing among five approaches (2nd ed.). Thousand Oaks: Sage.

De Villiers, M. R., De Villiers, P. J., \& Kent, A. P. (2005). The Delphi technique in health sciences education research. Medical Teacher, 27(7), 639-643.

Estes, J., \& Pierce, D. E. (2012). Pediatric therapists' perspectives on occupation-based practice. Scandinavian journal of occupational therapy, 19(1), 17-25.

Goldstein-Lohman, H., Kratz, A., \& Pierce, D. (2003). A study of occupation-based practice. In D. Pierce (Ed.), Occupation by design building therapeutic power (pp. 239-261). Philadelphia: F.A. Davis Company.

Golledge, J. (1998a). Distinguishing between occupation, purposeful activity and activity, part 1: Review and explanation. British Journal of Occupational Therapy, 61(3), 100-105.

Golledge, J. (1998b). 'Is there unnecessary duplications of skills between occupational therapist and physiotherapist?'. British Journal of Occupational Therapy, 61(4), 161-162.

Gray, J. M. (1998). Putting occupation into practice: Occupation as ends, occupation as means. American Journal of Occupational Therapy, 52(5), 354-364.

Hasson, F., Keeney, S., \& McKenna, H. (2000). Research guidelines for the Delphi survey technique. Journal of Advanced Nursing, 32(4), 1008-1015.

Jensen, G. M., Gwyer, J., \& Shepard, K. F. (2000). Expert practice in physical therapy. Physical Therapy, 80(1), 44-52.

Keeney, S., Hasson, F., \& McKenna, H. (2006). Consulting the oracle: Ten lessons from using the Delphi technique in nursing research. Journal of Advance Nursing 53(2), 205-212. 
Keeney, S., Hasson, F., \& McKenna, H. P. (2001). A critical review of the Delphi technique as a research methodology for nursing. International Journal of Nursing Studies, 38(2), 195-200.

Keilhofner, G. (2009). Conceptual foundation of occupational therapy practice (4th ed.). Philadelphia: F.A Davis.

Kuipers, K., \& Grice, J. W. (2009). The structure of novice and expert occupational therapists' clinical reasoning before and after exposure to a domain-specific protocol. Australian Occupational Therapy Journal, 56(6), 418-427.

Lee, S. W., Taylor, R., Kielhofner, G., \& Fisher, G. (2008). Theory use in practice: A national survey of therapists who use the Model of Human Occupation. American Journal of Occupational Therapy, 62(1), 106-117.

Malaysian Occupational Therapy Association. (2010). History of Malaysian Occupational Therapy Association. Retrieved 27 Nov 2014, 2011, from http://www.occupational-therapy.org.my/history.html\#

Mullersdorf, M., \& Ivarsson, A.-B. (2011). Occupation as described by academically skilled occupational therapists in Sweden: A Delphi study. Scandinavian Journal of Occupational Therapy, 18(2), 85-92.

Nilchaikovit, T., Hill, J. M., \& Holland, J. C. (1993). The effects of culture on illness behavior and medical care: Asian and American differences. General Hospital Psychiatry, 15(1), 41-50.

Pierce, D. (2001). Occupation by design: Dimensions, therapeutic power, and creative process. American Journal of Occupational Therapy, 55(3), 249-259.

Powell, C. (2003). The Delphi technique: Myths and realities. Journal of Advanced Nursing, 41(4), 376-382.

Rogers, S. (2007). Occupation-based intervention in medical-based settings. OT Practice, 12(15), 10-16.

Shinohara, K., Yamada, T., Kobayashi, N., \& Forsyth, K. (2012). The model of human occupation-based intervention for patients with stroke: A randomised trial. Hong Kong Journal of Occupational Therapy, 22(2), 60-69.

Stack, R., \& Barker, D. (2011). Students as advocates for occupation-based practice. Occupational Therapy Now, 13(3), 13-15.

Toth-Fejel, G., Toth-Fejel, G. F., \& Hedricks, C. (1998). Occupation-centered practice in hand rehabilitation using the Experience Sampling Method. American Journal of Occupational Therapy, 52(5), 381-385.

Trombly, C. A. (1995). Occupation: Purposefulness and meaningfulness as therapeutic mechanisms. American Journal of Occupational Therapy, 49(10), 960-972.

Unsworth, C. (2001). The clinical reasoning of novice and expert occupational therapists Scandinavian Journal of Occupational Therapy, 8, 163-173. 